\title{
Probabilistic models for risk assessment of disasters
}

\author{
A. Lepikhin \& I. Lepikhina \\ Department of Safety Engineering Systems, \\ SKTB "Nauka" KSC SB RAS, Russia
}

\begin{abstract}
This article considers risk models related to disasters of complex engineering systems. It is proposed to include parameters of system vulnerability, survivability and security into risk models. Types of risk function for different disaster mechanisms are discussed. It is shown that the risk functions may have both exponential and power mode peculiarity. The mechanism of transformation the exponential distribution into power mode (Pareto) distribution is determined. Keywords: engineering systems, disaster, risk function, probabilistic model, failure processes.
\end{abstract}

\section{Introduction}

Numerous studies have been carried out in recent years on safety of engineering systems and technologies. Safety factors, criteria, models and methods of risk assessment are permanently discussed in literature $[1,2]$. Based on analysis of theory and practices on safety measures for technical systems a number of problems in risk analysis methodology can be formulated [3].

The first problem is that technological progress provides the opportunity of making more and more complex and large systems. This in turn increases manmade hazards. Along with, the safety requirements are becoming stricter, while the securing resource base is limited. We think that we are now above the crisis point when the safety requirements exceed securing resources. In future, the gap will grow and the risks will increase. These new risks will be of critical and global type and must be investigated. The problem is that the main attention is paid for research of statistical events with small risks. At the present time, evolving critical systems are the main source of engineering hazards. These 
systems are deeply integrated in the life-supporting infrastructure. The disasters of the systems influence a great number of people and lead to large-scale hazards for natural environment. The tragic examples of this are the Deepwater Horizon and Sayano-Shyshenskaya HPP disasters.

The peculiarity of critical systems lies in their complicated structure and in self-organization quality they possess. These systems have an ability to convert the structure, to create new configurations and hazards during their life cycle. The recent investigations have shown that self-organization is moving to selforganizing criticality, under which the systems turn out to be extra sensitive to breakdowns and external influence. Another feature lies in fast evolution of critical systems, which takes the lead over the hazards exploration. As a result, disaster risks of critical systems are rapidly increased. The Deepwater Horizon and Sayano-Shyshenskaya HPP disasters have shown that we must investigate the critical risks.

The second problem is that the new risks are unique and their evaluation should be based on a new probabilistic paradigm. The statistical paradigm uses hazards and risk as the basic definitions. The probabilistic paradigm introduces the new basic definitions of risk theory, such as vulnerability, survivability and security (protection). These definitions are determined by the securing resources base and reflect the analyzed peculiarities of systems. Therefore, a new approach in risk theory should be considered. This approach must include assessment of vulnerability, survivability and security within the disaster risk evaluation.

The third problem consists in the need to develop new risk models of disasters. The new basic definitions must be included in models of risk assessment. Usually risk assessment is based on analysis of the disaster probability and loss probability. Along with the basic definitions, the new risk models must include vulnerability, survivability and security assessment. It is vital to develop a new methodology of risk assessment. The disasters are complicated events with hierarchical evolving structure. The level of risk depends on the object and the level of its structure being exposed to hazards. That is why methodology of hierarchical multiobjective risk analysis must be developed. Added to everything else disasters have different mechanisms of occurrence. Type of disaster determines the law of risk function. Therefore, we need to investigate the link between the disaster mechanism and type of risk function.

In this paper we propose new probabilistic models of disaster risk, which are outside the framework of traditional models $[1,2]$.

\section{Basic risk model (risk function)}

Disaster risk of engineering system is commonly associated with the appearance of an initial event. Initial events are usually results of human error, external influence or violation of technological processes. If a system is vulnerable to such factors, the initial event forms the core damages in the structure. Development of damages leads to changes in structure and system state at random time points. Reaching the critical level of damages results in the system 
disaster. Thus, risk function can be defined as a function of initial event and core damage event sequence

Risk $=$ function (Initial Event frequency $\times$ Core Damage Event sequences $)$

Damage development depends not only on system vulnerability but also on structure survivability and availability of systems of active and passive safety. This is why a risk function for evolving critical systems should be defined in the following form [3]

Risk $=$ function $($ Initial Event frequency $\times$ Vulnerability $\times$

Core Damage Event sequences $\times$ Survivability $\times$ Safety)

As already discussed, the distinctive feature of evolving systems is that there can take place random jumps of conditions with breakdown of previous and creation of new structures. Structural breakdowns in Large Scale, Super-Large Scale, Global Scale systems lead to cascaded events and nonlinear effects. In this case risk assessment of disasters can be done according to the model (2). Hereinafter formula (2) will be used as the basic. We will consider its structure, the probability assessment of initial events and sequence of damages.

\section{Exponential risk function}

Mathematical model of dynamic stochastic system with accidental structure can be presented in the following form:

$$
D(t)=A^{S}(D, t)+B^{S}(D, t) \xi(t)
$$

where $D(t)$ is n-dimensional vector of damages, $S(t)$ is structure number, $A, B$ are prescribed matrix arrays of states, $\xi$ is Gaussian noise with zero mean, $t$ is time. The vector of damages includes Multiple Material Element Damages and Multiple Elements Damages of structure.

Due to the existence of cross impact and interrelationship of disaster processes, risk models may be considered both in the space of damages $D$ and in the state space $S$. The choice is determined by dimensionality and complexity of problem formulation.

Risk models in the space of damages are considered for systems, which disasters occur due to damages of one or two components of structure. In this case, the disaster probability is determined in the following form:

$$
P_{f}(D, t)=P\left\{D(t) \geq D_{c}(t)\right\}
$$

where $D_{c}$ is critical level of damages.

According to the nature of process, the answer for (4) can be obtained on the basis of cumulative model or stochastic processes of crosses model. The peculiarities of such models have been discussed in details in the works $[3,4]$. 
Using these models the vulnerability, survivability and security of systems can be considered only towards to processes $D(t)$ and $D_{c}(t)$.

The greater interest is paid to disaster models of complicated hierarchical systems. In this case, the hierarchy of structural damages plays the main role. Thus, to formulate the risk model we need to turn to analysis of the state probability dynamics and disaster trajectory.

Let us observe the structural multistep disaster model (Fig. 1). Let us suppose that at the moment $t_{0}$ the system was in the state $S_{0}$ (normal operating state). As a result of influence with magnitude $\lambda$ from initial event at the moment $t_{\lambda}$ the system turns to the state $S_{1}$ (initial faulty state). Further the propagation of structural damages with consistent state, transition and formation of the disaster trajectory $\psi:\left\{S_{1}, S_{2}, \ldots S_{f}\right\}$ takes place.

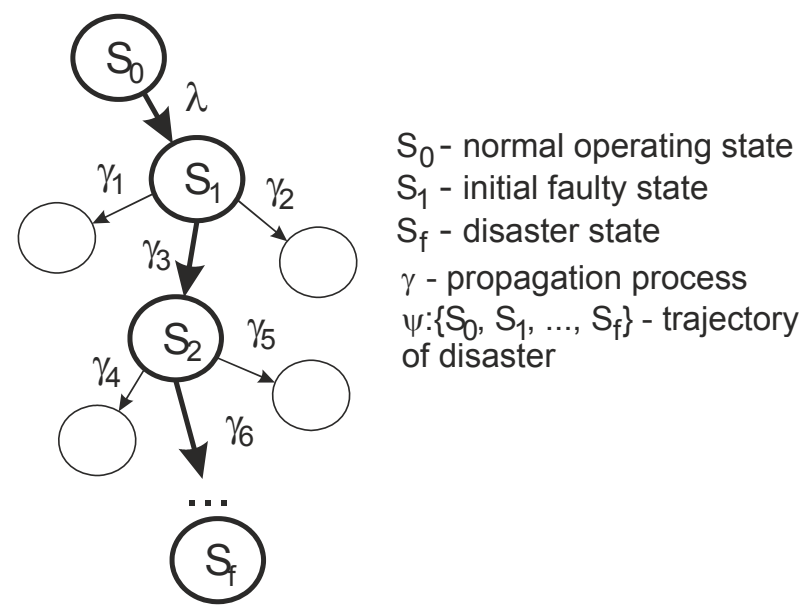

Figure 1: $\quad$ Notion of disaster trajectory.

Probability $P\left(S_{f}\right)$ of disaster (reaching the final state $S_{f}$ ) can be defined by:

$$
P\left(S_{f}\right)=\lambda P\left(S_{0}\right) \sum_{i} t_{S i}
$$

where $P\left(S_{0}\right)$ is probability of the normal operation state.

Magnitude of initial event is determined by methods of mathematical statistics or can be evaluated through the density function of probability distribution $f_{\lambda}(t)$ of waiting period for this event:

$$
\lambda=\left[\int_{0}^{\infty} t f_{\lambda}(t) d t\right]^{-1}
$$

It is commonly supposed that $f_{\lambda}(t)$ is of exponential type $f_{\lambda}(t)=\lambda \mathrm{e}^{-\lambda t}$.

The sum in equation (5) is defined by survivability and security of system structure. It can be calculated from the following equation: 
$t_{\Sigma}=\sum_{i} t_{S i}=\int_{0}^{\infty} \prod_{i} P_{i}(t) d t=$
$\int_{0}^{\infty} f_{\lambda}(t) \int_{t_{1}}^{\infty} f_{01}(t) \int_{t_{2}}^{\infty} f_{12}\left(t_{2}\right) \ldots \int_{t_{n}}^{\infty} f_{n-1, n}\left(t_{n}\right) d t_{n} \ldots d t_{1} d t$

Similarly to (5) we can obtain the equation for magnitude of state achievement:

$$
\Lambda\left(S_{f}\right)=\lambda P\left(S_{0}\right) \sum_{i} \lambda_{S i}
$$

Taking into account equation (8), the probability of disaster $P\left(S_{f}\right)$ can be expressed as:

$$
P\left(S_{f}, t\right)=1-\exp \left\{-\Lambda\left(S_{f}\right) t\right\}
$$

It is possible to show that in the examined scheme the processes of structural damages are precritical or critical, for which the extinction probability equals 1 . Assuming that the losses from damages are of additive form $u_{i}=u_{i-1}+\Delta u$, the accumulated total losses will concentrate near the average value. Due to the fact that function $f(t)$ in formula (7) and function (9) are of exponential type, the risk function will also have exponential peculiarity. Such risk functions are typical for disasters of Large Scale Systems.

\section{Power mode risk function}

Let us observe another scheme of damage propagation in a hierarchical structure. Let us take into account that a large number of elements in the system leads to a large number of interactions and nonlinear effects. We suppose that damaging processes simultaneously impact several subsystems (Fig. 2). In this case due to feedback coupling a self-organizing cluster of faulty states is generated. This cluster determines a destruction front, which consists of all the damage trajectories. Every damage in the cluster causes new failure processes. In this

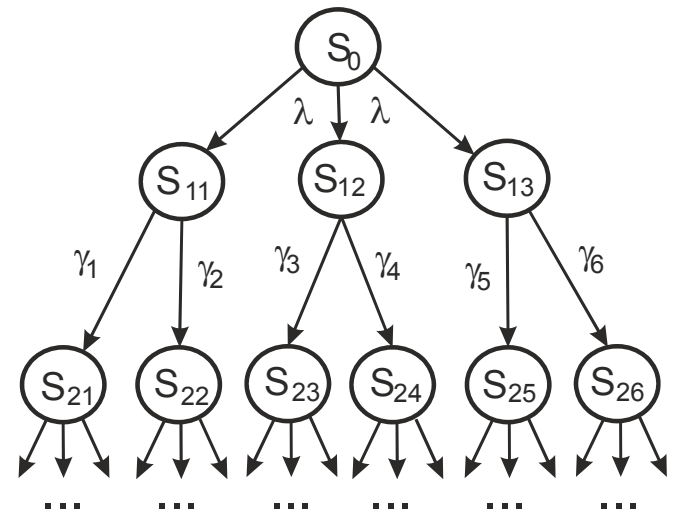

Figure 2: Multi-failure disaster trajectory. 
case the expected value of a number of new failure processes exceeds $1, \mathrm{M}[D(t)]$ $>1$. The process turned out to be above-critical, for which the extinction probability is less than 1 . As a result, cascaded damages with a typical multiplicative form of loss $u_{i}=(1+k) u_{i-1}, k>1$ are generated. The final value of losses can be very large as the average loss value tends to infinity. Consequently, the risk function has "heavy tail" instead of exponential form (Fig. 3).

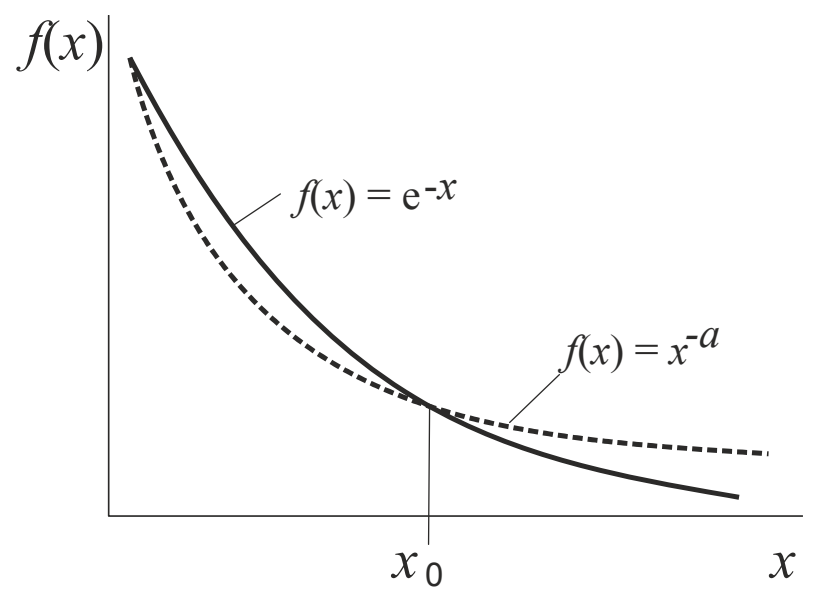

Figure 3: $\quad$ Probability risk function for exponential and power mode law.

In literature for description of such "tails" the Pareto distribution is usually used [5]

$$
F(x)=1-\left(x_{0} / x\right)^{a}
$$

where $x_{0}, a$ are parameters of distribution.

For a certain range of parameters the exponential distribution and Pareto distribution closely approach or pass into each other at the point $x_{0}$. If $x<x_{0}$, the risk function is exponential, while at $x \geq x_{0}$, the function is of power mode (Pareto) type. If the expected values of the distributions are equal, parameter $a$ is related to parameter $\lambda$ as $a=\lambda+1$.

The Pareto distribution in the disaster risk function appears as result of nonlinear transformation of physical processes of structural damages to the processes of loss formation. These transformations can be presented by the formula relating the parameters $\lambda$ and $a$. One form of such transformations is as following [6]

$$
a=\frac{z(\lambda+1)}{z(\lambda+1)-1}, z>1
$$

The parameter $z$ reflects the transformation of the heaviness of system damage. As an example in the Fig. 4 the nonlinear transformation (11) of the exponential risk function into Pareto distribution is presented. As we can see, the probability of large losses increases with the growth of damage severity. 


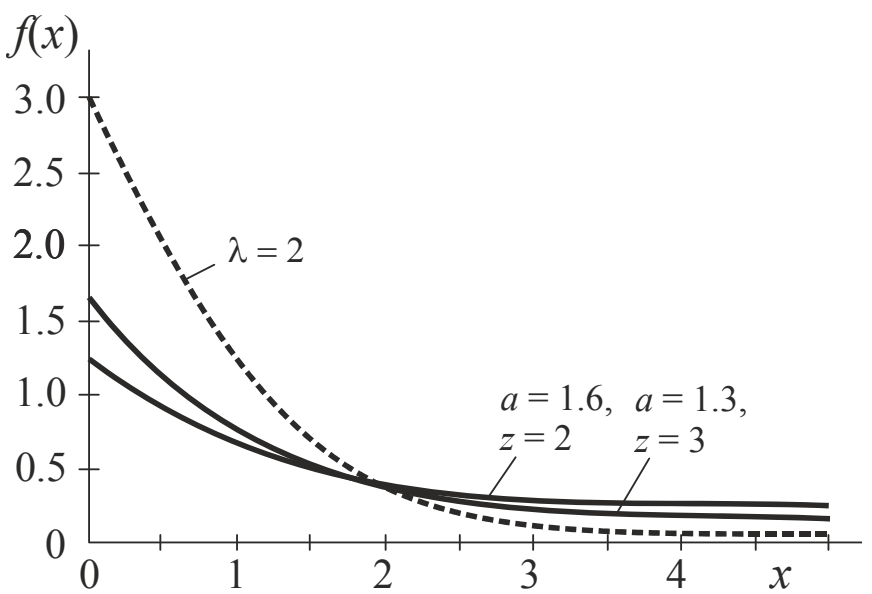

Figure 4: Nonlinear transformation of risk function.

There are several critical values of parameter $a$ in the Pareto distribution. The most interesting processes take place when $a \leq 2$. In other words, when the distribution variance tends to infinity. If $a \leq 1$, the expected value of the distribution approaches infinity. When $a \approx 1$, the processes of self-organizing criticality occur in complex systems.

The considered peculiarities are typical for disaster risk functions of SuperLarge Scale and Global Scale systems.

\section{Conclusion}

Modern society can be considered as society of risk. This risk is determined not only by natural hazards but also by growing hazards of engineering disasters. Nowadays, the greatest danger goes from evolving critical systems that are the basis of life-supporting infrastructure. Developing the probabilistic paradigm and probabilistic risk models is required to analyze disasters of such systems. Probabilistic risk models should take into account vulnerability, survivability and safety of complex systems in relation to disastrous external influences and internal damage processes.

In most cases disaster risk models are based on the use of probability distribution laws of exponential type. These laws adequately describe a damage distribution in complex systems. However, they become unsatisfactory when we analyze not the damage processes, but the results of their impact on systems. In this case the use of power mode law of probability distribution (Pareto's law) is more relevant in modeling the risk of disasters. Further study on application of the Pareto distribution for evolving critical systems will allow predicting the possible critical states. This in turn will help in developing measures to avoid such critical states. Our results show the need for excluding initial events and avalanche of damages to provide the safety of evolving critical systems. The great attention should be paid to research on self-organizing criticality. 
90 Safety and Security Engineering IV

\section{References}

[1] Perrow Ch. Normal Accidents: Living with High-Risk Technologies, N.Y.: Basic Books, 386 pp., 1999.

[2] Henley E., Kumamoto H. Probabilistic risk assessment and management for engineers and Scientists. IEEE PRESS, 597 pp., 1996.

[3] Lepikhin A. A Critical Look at Risk Analysis of Disasters. Risk Analysis VII \& Brownfields V. WIT Press, pp. PI-59-PI-67, 2010.

[4] Lepikhin A., Moskvichev V., Cherniaev A. Risk analysis of the technical systems, Novosibirsk: Nauka, 173 pp., 2003.

[5] Malinetskiy G.G., Potapov A.B. Contemporary problems of the non-linear dynamics, M.: Editorial URSS, 360 pp., 2002.

[6] Shyshkin V.M. Power mode distribution and risk management of critical systems. Writings ISA RAS, Vol. 31, pp. 42-63, 2007. 